\title{
APLICACIÓN DEL MÉTODO DE FERTILIZACIÓN RAZONADA A UN CULTIVO DE PEONIAS*
}

\section{APPLICATION OF THE RATIONAL FERTILIZATION METHOD TO A PEONY CROP}

Sáez, M. C.

Universidad de Magallanes. Casilla 113-D, Punta Arenas, Chile.

E-mail: consuelo.saez@umag.cl

\section{INTRODUCCION}

Entre los aspectos más importantes que involucra el cultivo de la peonía herbácea, la fertilización es tal vez uno de los puntos más condicionantes en la obtención de un mayor número de flores de óptima calidad. Sin embargo, las investigaciones sobre este cultivo en Chile son muy escasas y los resultados obtenidos a través de experimentación en el extranjero no siempre son aplicables a las condiciones nacionales, por las diferencias tanto de clima como de suelo en cada zona donde se ha introducido este cultivo (Pinochet 1999). Por esta razón, para el cálculo de la dosis de fertilizantes se ha utilizado el método del balance nutricional, propuesto por Standford en 1973 y desarrollado en Chile por Rodríguez (1993). En dicho método, la fertilización de los cultivos se enmarca dentro de las interacciones del subsistema ecológico (suelos, clima, disponibilidad de nutrientes, etc.) con el subsistema de cultivos (especie, historial de fertilización, residuos, etc.) que se encuentra en un determinado ámbito socio-económico (agroecosistemas) y que condicionan la expresión genética del potencial productivo. La biomasa alcanzable del cultivo en un delimitado agroecosistema genera una demanda de nutrientes para satisfacer sus necesidades metabólicas. Esta demanda, en especial de N, P y K, no es satisfecha con el suministro de nutrientes que es capaz de entregar el suelo y se produce un déficit nutricional que debe ser solucionado a través de la fertilización (Rodríguez 1993, Pinochet 1999). Dentro de este contexto, para la aplicación del modelo de fertilización razonada, el objetivo del presente trabajo fue deter- minar la demanda de nutrientes a través de la obtención de los parámetros que la componen: rendimiento esperado (RE), índice de cosecha (IC) y requerimientos internos (RI) de cada nutriente en el cultivo de la peonía herbácea.

\section{MATERIALES Y METODOS}

Para la determinación de los parámetros RE, RI e IC se cosecharon 10 varas comerciales por repetición desde cuatro predios con diferentes realidades edafoclimáticas (Villa Don Miguel, Osvaldito, Leñadura, Universidad de Magallanes), obtenidas durante la cosecha de la temporada 2001/2002. Las variedades muestreadas fueron Top Bras y Sarah Bernhardt (Paeonia lactiflora $x$ P. lactiflora), Red Charm (P. lactiflora $x$ P. officinallis) y Flame (P. lactiflora $x P$. peregrina). En total fueron cosechadas 480 varas. Estas muestras, fueron secadas a $70^{\circ} \mathrm{C}$ en una estufa de ventilación forzada y enviadas al Laboratorio de Análisis de Suelos y Foliar de la Facultad de Agronomía de la Pontificia Universidad Católica de Chile.

\section{RESULTADOS Y CONCLUSIONES}

Los análisis de laboratorio indicaron una absoluta homogeneidad de valores tanto para los distintos predios como para los distintos tipos de híbridos y por esta razón los resultados son presentados en base a promedios. El rendimiento esperado (RE) para cada agroecosistema fue estimado en base al conocimiento de las condiciones edafoclimáticas y de manejo. Para las condiciones de Magallanes, el RE es de 3, 5 y 10 varas comerciales por planta, a la tercera, 
Cuadro 1. Rendimiento esperado (RE) expresado en KgMS ha ${ }^{-1}$ a distintas edades (temporadas $3^{\circ}, 4^{\circ}$ y $5^{\circ}$ desde la plantación) y densidades de plantación ( $\mathrm{N}^{\circ}$ plantas ha $\left.{ }^{-1}\right)$.

Table 1. Expected yield expressed in KgDMS ha ${ }^{-1}$ at different ages (3rd, 4th and 5th season after plantation) and plantation densities $\left(\mathrm{N}^{\circ}\right.$ plants $\left.\mathrm{ha}^{-1}\right)$.

\begin{tabular}{cccc}
\hline $\begin{array}{c}\text { TEMPORADA } \\
N^{\circ} \text { varas }\end{array}$ & & & \\
$\begin{array}{c}\text { comerciales/planta } \\
N^{\circ} \text { plantas ha }\end{array}$ & $\begin{array}{c}3^{\circ} / 3 \\
\mathrm{KgMS} \mathrm{ha}^{-1}\end{array}$ & $\begin{array}{c}4^{\circ} / 5 \\
\mathrm{KgMS} \mathrm{ha}^{-1}\end{array}$ & $\begin{array}{c}5^{\circ} \mathrm{a} 12^{\circ} / 1 \mathrm{C} \\
\mathrm{KgMS} \mathrm{ha}^{-1}\end{array}$ \\
\hline 10.000 & 870 & 1.450 & 2.900 \\
15.000 & 1.305 & 2.175 & 4.350 \\
20.000 & 1.740 & 2.900 & 5.800 \\
25.000 & 2.175 & 3.625 & 7.250 \\
\hline
\end{tabular}

Cuadro 2. Requerimientos internos (RI) de macro y microelementos para peonías.

Table 2. Internal requirements (IR), of macro and microelements for peonies.

\begin{tabular}{cc}
\hline ELEMENTO & REQUERIMIENTO INTERNO (RI) \\
\hline MACRONUTRIENTES & \\
PRIMARIOS & $\%$ \\
Nitrógeno (N) & 1.41 \\
Fósforo (P) & 0.24 \\
Potasio (K) & 1.37 \\
SECUNDARIOS & \\
Calcio (Ca) & 1.31 \\
Magnesio (Mg) & 0.27 \\
Azufre (S) & 0.20 \\
MICRONUTRIENTES & $\mathrm{ppm}$ \\
Cobre (Cu) & 5 \\
Zinc (Zn) & 33 \\
Manganeso (Mn) & 20 \\
Boro (B) & 29 \\
\hline
\end{tabular}

cuarta y desde la quinta temporada (desde la plantación) respectivamente (Cuadro 1).

En el Cuadro 2 se presentan los requerimientos internos de cada nutriente por parte del cultivo de peonías, estos resultados representan el contenido en porcentaje de cada elemento en los tejidos vegetales y junto con la determinación del rendimiento esperado por los productores en las distintas realidades edafoclimáticas del país dan origen a las dosis de fertilizantes para cada predio.

A su vez, el índice de cosecha (IC) representa la proporción entre el rendimiento esperado y la biomasa a partir de la cual la planta de peonía genera la producción de la temporada siguiente.
Para una plantación adulta con un promedio de 10 varas comerciales por planta, el índice de cosecha es de $50 \%$, es decir para cosecha 10 varas comerciales/planta, ésta debe tener al menos 20 varas en total.

\section{BIBLIOGRAFIA}

PINOCHET, D. 1999. Fertilización de plantas bulbosas. In: Seemann, P.; Andrade, N. (eds.) Cultivo y manejo de plantas bulbosas ornamentales. Universidad Austral de Chile, Facultad de Ciencias Agrarias, pp. 123-137.

RODRIGUEZ, S.J. 1993. La fertilización de los cultivos, un método racional. Colección en Agricultura. Facultad de Agronomía, Pontificia Universidad Católica de Chile. Santiago, Chile. 291 p.* 